\title{
Global parameter-space correlations of coherent searches for continuous gravitational waves
}

\author{
Reinhard Prix and Yousuke Itoh \\ Max Planck Institut für Gravitationsphysik, Albert Einstein Institut, Am Mühlenberg 1, \\ Golm 14476, Germany \\ E-mail: Reinhard.Prix@aei.mpg.de
}

Received 31 March 2005, in final form 8 June 2005

Published 6 September 2005

Online at stacks.iop.org/CQG/22/S1003

\begin{abstract}
The space of phase parameters (sky position, frequency, spindowns) of a coherent matched-filtering search for continuous gravitational waves from isolated neutron stars shows strong global correlations ('circles in the sky'). In the local limit this can be analysed in terms of a parameter-space metric, but the global properties are less well studied. In this work, we report on our recent progress in understanding these global correlations analytically for short to intermediate (less than a month, say) observation times and neglecting spindowns. The location of these correlation circles in parameter space is found to be determined mostly by the orbital velocity of the earth, while the spin motion of the detector and the antenna patterns only contribute significantly to the amplitude of the detection statistic along these circles.
\end{abstract}

PACS numbers: $04.80 . \mathrm{Nn}, 95.75 .-\mathrm{z}$

(Some figures in this article are in colour only in the electronic version)

\section{Introduction}

Continuous gravitational waves from, for example, rotating neutron stars with nonaxisymmetric deformations such as mountains or oscillation modes, are one of the primary targets of current-generation detectors of both interferometric (e.g. see [2, 3]) as well as bar-detector design [9].

In this paper, we restrict ourselves to gravitational waves emitted from isolated neutron stars with negligible proper motion, for which the signal can be assumed to be a nearly monochromatic sinusoid (with slowly decreasing frequency) in the solar-system barycentre frame (SSB). The corresponding signal received at the detector will be Doppler modulated by the spin of the earth and its orbital motion around the sun. The phase of the received signal therefore depends not only on its intrinsic frequency $f$ and spindowns $f^{(k)} \equiv \mathrm{d}^{k} f / \mathrm{d} t^{k}$, but also 
on the sky position (denoted by $\alpha$ and $\delta$ for right ascension and declination) of the source and on the detector location. In addition there is a time-dependent amplitude modulation of the signal depending on its polarization angle $\psi$ and amplitudes $h_{+}$and $h_{\times}$. However, as shown in [5], one can eliminate these 'amplitude parameters' together with the initial phase of the signal $\Phi_{0}$ by analytically maximizing the detection statistic over these parameters. The resulting reduced parameter space therefore only consists of the 'phase parameters' $\mathcal{P}=\left\{\alpha, \delta, f, f^{(k)}\right\}$, and the corresponding partially maximized detection statistic is usually referred to as the ' $\mathcal{F}$-statistic'.

Given a stretch of data $x(t)$ from the detector, an untargeted search (as opposed to a search for known pulsars) consists of calculating the detection statistic $\mathcal{F}$ over the parameter space (by sampling individual points) and determining the location of 'candidates' that cross a predetermined threshold. For a given signal $\mathcal{P}_{\mathrm{s}}=\left\{\alpha_{\mathrm{s}}, \delta_{\mathrm{s}}, f_{\mathrm{s}}, f_{\mathrm{s}}^{(k)}\right\}$, the detection statistic $\mathcal{F}$ in a search-point $\mathcal{P}$ generally depends on the parameter mismatch $\Delta \mathcal{P} \equiv \mathcal{P}_{\mathrm{s}}-\mathcal{P}$, i.e., $\mathcal{F}=\mathcal{F}\left(\mathcal{P}_{\mathrm{s}}, \Delta \mathcal{P}\right)$ (strictly speaking, $\mathcal{F}$ also depends on the amplitude parameters of the signal). Neglecting noise, one can expect $\mathcal{F}$ to be maximal for the perfectly matched searchpoint $\Delta \mathcal{P}=0$. In the local neighbourhood of this point, the loss $\Delta \mathcal{F} \equiv \mathcal{F}\left(\mathcal{P}_{\mathrm{s}}, 0\right)-\mathcal{F}\left(\mathcal{P}_{\mathrm{s}}, \Delta \mathcal{P}\right)$ in detection statistic can be quantified in terms of a parameter-space metric as discussed in $[8,4]$. Namely, neglecting effects of the amplitude parameters of the signal, this metric can be defined as $\Delta \mathcal{F}\left(\mathcal{P}_{\mathrm{s}}, \Delta \mathcal{P}\right) / \mathcal{F}\left(\mathcal{P}_{\mathrm{s}}, 0\right)=\sum_{i, j} g_{i j}\left(\mathcal{P}_{\mathrm{s}}\right) \Delta \mathcal{P}^{i} \Delta \mathcal{P}^{j}+\mathcal{O}\left(\Delta \mathcal{P}^{3}\right)$.

In the case of isolated neutron stars, we find that this metric is highly anisotropic in terms of the above parameter-space variables (in particular with respect to sky position), as will also be seen in section 6. Furthermore, the global behaviour of $\mathcal{F}$ differs dramatically from the local quadratic decrease as a function of parameter mismatch $\Delta \mathcal{P}$. This can be seen, for example, in figure 2 of an all-sky search at fixed frequency for an injected signal. For each target search frequency $f$ within the Doppler window of the signal frequency $f_{\mathrm{s}}$, one finds a very thin circular band on the sky along which $\mathcal{F}$ is of comparable magnitude to its maximum, while it drops sharply to zero in the directions orthogonal to this 'circle'. In the local limit, this feature has already been pointed out in [7].

In this work, we present a first approximate analytic description of the location of these 'circles in the sky', identifying their dominant physical origin with the Doppler shift due to the orbital motion around the sun. The Doppler shift induced by the spin motion of the earth as well as the amplitude modulation due to the rotating antenna pattern is found to give only very small corrections to the structure of these patterns in parameter space (i.e., the location of the circles), while they do contribute significantly to the amplitude of the $\mathcal{F}$-statistic along these circles. Note that, contrary to the global structure, the width of these circles is expected to be well described by the local metric, but we postpone a detailed study of this aspect to future work.

\section{Matched filtering of continuous signals}

The gravitational-wave strain $h(t)$ at the detector can be written in the form

$$
h(t)=F_{+}(t) h_{+}(t)+F_{\times}(t) h_{\times}(t)
$$

where $F_{+, \times}$are the antenna-pattern functions and $h_{+, \times}$are the two polarization components of the gravitational wave. For a continuous pulsar signal we can write

$$
h_{+}(t)=A_{+} \sin \left(\Phi(t)+\Phi_{0}\right), \quad h_{\times}(t)=A_{\times} \cos \left(\Phi(t)+\Phi_{0}\right)
$$


The phase $\Phi(t)$ of the signal is assumed to be of the form

$$
\Phi\left(t ; f^{(k)}, \vec{n}\right)=2 \pi \sum_{k=0}^{s} \frac{f^{(k)}}{(k+1) !} \tau^{k+1}(t, \vec{n}),
$$

where $s$ is the number of spindown parameters and $\tau$ is the arrival time in the solar-system barycentre (SSB) of a wave front from direction $\vec{n}=(\cos \delta \cos \alpha, \cos \delta \sin \alpha, \sin \delta)$ arriving at the detector at time $t$, i.e., (neglecting small relativistic effects)

$$
\tau(t, \vec{n}) \equiv t+\frac{\vec{r}(t) \cdot \vec{n}}{c},
$$

with $\vec{r}(t)$ denoting the vector from the SSB to the detector. As shown in [5], the full phase model (3) can be simplified by an approximate model without significant loss in detection statistic, namely

$$
\Phi(t) \approx 2 \pi\left[\sum_{k=0} \frac{f^{(k)}}{(k+1) !} t^{k+1}+\frac{\vec{r}(t) \cdot \vec{n}}{c} \sum_{k=0} \frac{f^{(k)}}{k !} t^{k}\right],
$$

which will be dominated by the first term, i.e., $\Phi(t) \approx 2 \pi f t$. In the case of stationary Gaussian noise, the likelihood ratio $\Lambda$ (which is the optimal detection statistic in the sense of Neyman-Pearson) is given by

$$
\ln \Lambda=\frac{T}{S_{h}}\left[(x \| h)-\frac{1}{2}(h \| h)\right],
$$

where $S_{h}$ is the power spectral density of the noise, and the scalar product $(x \| y)$ is defined as

$$
(x \| y) \equiv \frac{2}{T} \int_{0}^{T} x(t) y(t) \mathrm{d} t .
$$

\section{Simplified matched-filtering statistic: neglecting amplitude modulations}

In the following, we make the simplifying assumption that we can neglect the amplitude modulation caused by the antenna pattern, so we assume $F_{+, x} \approx$ const, and using (1) and (2) this results in the simplified strain model

$$
h(t)=A_{1} \cos \Phi(t)+A_{2} \sin \Phi(t) .
$$

Maximizing the log-likelihood function (6) over the two unknown amplitudes $A_{1,2}$, we obtain

$$
\ln \Lambda_{\mathrm{ML}}=\frac{T}{2 S_{h}}\left|\left(x \| \mathrm{e}^{-\mathrm{i} \Phi}\right)\right|^{2} \equiv \frac{T}{2 S_{h}}|X|^{2},
$$

defining the matched-filtering amplitude $X$. In the following, we assume the data contain only a unit-amplitude pulsar signal of the form

$$
x(t)=\operatorname{Re}[s(t)] \quad \text { with } \quad s(t)=\exp \left[\mathrm{i} \Phi\left(t ; f_{\mathrm{s}}^{(k)}, \vec{n}_{\mathrm{s}}\right)\right],
$$

characterized by the signal phase parameters $f_{\mathrm{s}}^{(k)}$ and $\vec{n}_{\mathrm{s}}$. The matched-filtering amplitude $X$, defined in (9), can therefore be expressed as

$$
X=\left(x \| \mathrm{e}^{-\mathrm{i} \Phi}\right) \approx \frac{1}{T} \int s(t) \mathrm{e}^{-\mathrm{i} \Phi(t)} \mathrm{d} t=\frac{1}{T} \int \mathrm{e}^{\mathrm{i} \Delta \Phi(t)} \mathrm{d} t,
$$

where $\Delta \Phi$ is the phase-difference $\Delta \Phi(t)=\Phi_{\mathrm{S}}(t)-\Phi(t)$, and where we have used the fact that $\int \exp \left[\mathrm{i} 2 \pi\left(f+f_{\mathrm{s}}\right) t\right] \mathrm{d} t \approx 0$. We see from (11) that $|X|$ will have a global maximum of $|X|=1$ if the phase parameters of the template are perfectly matched to the signal, i.e., $\Delta \Phi=0$, while $|X|$ will decrease very rapidly for increasing phase mismatches.

The central aim of the following investigation is to determine the regions in parameter space for which the detection statistic $|X|$ is of order unity for a given signal $\left\{\vec{n}_{\mathrm{s}}, f_{\mathrm{s}}^{(k)}\right\}$. 


\section{Analytic approximation for $|X|^{2}$}

In order to simplify the following discussion, we restrict ourselves in this study to pulsar signals with negligible spindown over the observation period $T$, so we assume $f^{(k)} \approx 0$ for $k>0$. Using (5), we can therefore write the phase mismatch $\Delta \Phi(t)$ as

$$
\Delta \Phi\left(t ; \vec{n}_{\mathrm{s}}, f_{\mathrm{s}} ; \vec{n}, f\right)=2 \pi\left[\Delta f t+\frac{\vec{r}(t)}{c} \cdot \vec{A}\right],
$$

where we defined

$$
\Delta f \equiv f_{\mathrm{s}}-f \quad \text { and } \quad \vec{A} \equiv f_{\mathrm{s}} \vec{n}_{\mathrm{s}}-f \vec{n} .
$$

We can decompose the motion of the detector $\vec{r}(t)$ into its orbital and spin components, namely, $\vec{r}(t)=\vec{r}_{\text {orb }}(t)+\vec{r}_{\text {spin }}(t)$, where $\vec{r}_{\text {orb }}$ is the vector from the SSB to the centre of the earth and $\vec{r}_{\text {spin }}$ is the vector from there to the detector. Correspondingly, we have

$$
\Delta \Phi(t)=2 \pi \Delta f t+\Delta \Phi_{\mathrm{orb}}(t)+\Delta \Phi_{\mathrm{spin}}(t)
$$

where

$$
\Delta \Phi_{\text {orb }}=\frac{2 \pi}{c} \vec{r}_{\text {orb }}(t) \cdot \vec{A} \quad \text { and } \quad \Delta \Phi_{\text {spin }}=\frac{2 \pi}{c} \vec{r}_{\text {spin }}(t) \cdot \vec{A}
$$

\subsection{Spin motion of the earth}

Except for very short observation times $T \ll 1$ day, which are not relevant for searches for continuous waves, the phase modulation $\Phi_{\text {spin }}$ due to the spin motion of the earth is oscillatory, and can therefore not be treated using a Taylor expansion. However, following an approach first used in $[6,10]$, it is more advantageous to use the Jacobi-Anger expansion, which allows one to expand an oscillatory exponent in terms of the Bessel functions $J_{n}(z)$, namely

$$
\mathrm{e}^{\mathrm{i} z \cos \zeta}=\sum_{n=-\infty}^{\infty} \mathrm{i}^{n} J_{n}(z) \mathrm{e}^{\mathrm{i} n \zeta}
$$

In order to be able to use this, we write the detector motion $\vec{r}_{\text {spin }}(t)$ as

$$
\vec{r}_{\text {spin }}(t)=\sin \lambda R_{\oplus} \hat{z}+\cos \lambda R_{\oplus} \hat{\oplus}(t),
$$

where $\lambda$ is the latitude of the detector, $R_{\oplus}$ is the radius of the earth, $\hat{z}$ is the unit vector along the rotation axis and $\hat{\omega}(t)$ is the time-dependent unit vector orthogonal to $\hat{z}$, pointing from the rotation axis to the detector. Using this decomposition, we can write the phase mismatch due to the spin motion of the earth as

$$
\Delta \Phi_{\text {spin }}(t)=\Delta \Phi_{z}+K_{A} \cos \varphi(t)
$$

where we defined

$$
\Delta \Phi_{z} \equiv 2 \pi \frac{R_{\oplus}}{c} \sin \lambda A_{z}, \quad K_{A} \equiv 2 \pi \frac{R_{\oplus}}{c} \cos \lambda A_{\perp},
$$

and

$$
\varphi(t)=\varphi_{A}+\Omega_{\text {spin }} t \quad \text { with } \quad \varphi_{A}=\measuredangle\left(\vec{A}_{\perp}, \hat{\omega}(0)\right),
$$

and where $A_{z}$ and $\vec{A}_{\perp}$ are the parallel and orthogonal projections of $\vec{A}$ with respect to the rotation axis $\hat{z}$. Using this decomposition, we can apply the Jacobi-Anger expansion (16) as follows:

$$
\mathrm{e}^{\mathrm{i} \Delta \Phi_{\text {spin }}(t)}=\mathrm{e}^{\mathrm{i} \Delta \Phi_{z}} \sum_{n=-\infty}^{\infty} \mathrm{i}^{n} \mathrm{e}^{\mathrm{i} i \varphi_{A}} J_{n}\left(K_{A}\right) \mathrm{e}^{\mathrm{i} i \Omega_{\text {spin }} t} .
$$


Substituting this together with (14) into the matched-filtering amplitude (11), we obtain

$$
X=\mathrm{e}^{\mathrm{i} \Delta \Phi_{z}} \sum_{n=-\infty}^{\infty} \mathrm{i}^{n} \mathrm{e}^{\mathrm{i} n \varphi_{A}} J_{n}\left(K_{A}\right) Y_{n},
$$

where

$$
Y_{n}=\frac{1}{T} \int \exp \left[\mathrm{i}\left(2 \pi \Delta f t+\Delta \Phi_{\mathrm{orb}}(t)+n \Omega_{\mathrm{spin}} t\right)\right] \mathrm{d} t
$$

\subsection{Orbital motion around the sun}

Contrary to the spin motion, the orbital motion induces a slowly varying and non-oscillatory phase correction for observation times $T \ll 1$ year, so we can expand the orbital phasemismatch $\Delta \Phi_{\text {orb }}(t)$ in terms of the small quantity $\varepsilon \equiv \Omega_{\text {orb }} T \ll 1$, which leads to

$$
Y_{n}=\mathrm{e}^{\mathrm{i} \Delta \Phi_{\mathrm{orb}}(0)} \frac{1}{T} \int \exp \left[\mathrm{i}\left(a_{1} t+\sum_{k=2} a_{k} t^{k}\right)\right] \mathrm{d} t,
$$

with the coefficients

$$
\begin{aligned}
& \frac{a_{1}}{2 \pi}=\Delta f+\vec{\beta}(0) \cdot \vec{A}+n f_{\mathrm{spin}}, \\
& \frac{a_{k}}{2 \pi}=\frac{1}{k !} \vec{\beta}^{(k-1)}(0) \cdot \vec{A}, \quad(k>1),
\end{aligned}
$$

where we defined $f_{\text {spin }} \equiv \Omega_{\text {spin }} / 2 \pi$ and where $\vec{\beta}(t) \equiv \vec{V}(t) / c=\dot{\vec{r}}_{\text {orb }}(t) / c$ denotes the orbital velocity, and $t=0$ is the start of the observation.

Note that the integrals $Y_{n}$ will be strongly peaked as a function of the index $n$, namely by considering

$$
\left|Y_{n}\right| \approx\left|\frac{1}{T} \int \mathrm{e}^{\mathrm{i} a_{1} t} \mathrm{~d} t\right| \sim \operatorname{sinc}\left[a_{1} \frac{T}{2}\right]
$$

we see that $Y_{n}$ will have a maximum for the index $n^{*}$ closest to the point $a_{1}=0$, i.e.,

$$
n^{*}=-\operatorname{round}\left[\frac{\Delta f+\vec{\beta}(0) \cdot \vec{A}}{f_{\text {spin }}}\right]
$$

where the 'round $(x)$ ' rounds a real number $x$ to the nearest integer. As a first approximation, we therefore only keep the dominant term $n^{*}$ in the Jacobi-Anger expansion, i.e., we set $Y_{n} \approx Y_{n *} \delta_{n, n *}$, and so we obtain for the matched-filtering amplitude

$$
X \approx \mathrm{e}^{\mathrm{i}\left(\Delta \Phi_{z}+n^{*} \varphi_{A}\right)} \mathrm{i}^{n *} J_{n *}\left(K_{A}\right) Y_{n *} .
$$

We are interested in the regions of parameter space where $|X| \sim \mathcal{O}(1)$. In these regions, we can assume the exponent in (24) to be small, and therefore, after Taylor expanding and integrating (24), we can obtain the approximate expression

$$
|X|^{2} \approx J_{n *}^{2}\left(K_{A}\right)\left[1-\frac{\hat{a}_{1}^{2}}{12} T^{2}-\frac{\hat{a}_{1} a_{2}}{6} T^{3}+\mathcal{O}\left(T^{4}\right)\right] \equiv\left|X_{1}\right|^{2},
$$

where $\hat{a}_{1} \equiv a_{1}\left(n^{*}\right)$. 


\section{The 'maximum structure' of $|X|$}

One can see from its definition (11) that $|X|$ has a global maximum of $|X|=1$ in the perfectly matched case ( $f=f_{\mathrm{s}}$ and $\vec{n}=\vec{n}_{\mathrm{s}}$ ), while it will be nearly zero almost everywhere else, because generally $\Delta \Phi$ will not be small. Guided by the observed structure of the detection statistic (see section 6 , for example), we focus on the points in parameter space where $|X|$ (or $\mathcal{F}$ ) has a local maximum in target-frequency $f$ for a fixed sky-position $\vec{n}$, i.e.,

$$
\left.\frac{\partial|X|^{2}}{\partial f}\right|_{\vec{n}}=0 .
$$

In order to understand the qualitative features of these local maxima, we now also neglect the spin motion of the earth (setting $n^{*}=0$ and $K_{A}=0$ ), and using (29) and (25) we obtain the following condition for local maxima (in the sense of (30)):

$$
a_{1} a_{1}^{\prime}+a_{2} T a_{1}^{\prime}+a_{1} T a_{2}^{\prime}=0
$$

where we write $a_{k}^{\prime} \equiv \partial_{f} a_{k}(f, \vec{n})$. We can solve this order by order in $T$ (strictly speaking, in the small parameter $\varepsilon=\Omega_{\mathrm{orb}} T$ ), and to zeroth order we find (noting that $a_{1}^{\prime}=\mathcal{O}(1)$ ):

$$
\mathcal{O}\left(T^{0}\right): \quad a_{1} a_{1}^{\prime}=0 \quad \Longrightarrow \quad \Delta f+\vec{\beta}_{0} \cdot \vec{A}=0,
$$

where $\vec{\beta}_{0} \equiv \vec{\beta}(0)$. For the next order we therefore solve (31) with the ansatz $a_{1}=\lambda T$ (with $\lambda=\mathcal{O}(1))$, so we find

$$
\mathcal{O}(T): \quad a_{1}+a_{2} T=0 \quad \Longrightarrow \quad \Delta f+\vec{\beta}_{1} \cdot \vec{A}=0,
$$

where $\vec{\beta}_{1} \equiv \vec{\beta}(0)+\dot{\vec{\beta}}(0) \frac{T}{2}$ is the first-order approximation of the orbital velocity at the midpoint of the observation time. This scheme can be extended to arbitrary order in $T$, but for the qualitative discussion here we restrict ourselves to first order. The condition (33) can be expressed more explicitly as

$$
f\left(1+\vec{\beta}_{1} \cdot \vec{n}\right)=f_{\mathrm{s}}\left(1+\vec{\beta}_{1} \cdot \vec{n}_{\mathrm{s}}\right) .
$$

For a given signal $\left\{\vec{n}_{\mathrm{s}}, f_{\mathrm{s}}\right\}$, this equation describes the (approximate) location of the local maxima of $|X|$ as a function of $f$ for fixed $\vec{n}$. Conversely, this can be regarded at fixed frequency $f$, describing the sky-locations $\vec{n}$ in which the maximum occurs at this particular frequency $f$. From the above equation one can easily see that these points $\vec{n}$ describe a circle in the sky, which has its centre in $\vec{\beta}_{1}$, and an opening angle determined by the signal location and the target frequency. In the case $f=f_{\mathrm{s}}$, we see that $\vec{n}=\vec{n}_{\mathrm{s}}$ is one of the possible solutions of (34), and so the circle will pass through the signal location in the sky. If $f<f_{\mathrm{s}}$, then we must have $\vec{\beta}_{1} \cdot \vec{n}>\vec{\beta}_{1} \cdot \vec{n}_{\mathrm{s}}$, i.e., the opening angle of the circle has to be larger than in the case of a perfectly matched target frequency, and vice versa for the case of $f>f_{\mathrm{s}}$. For a $10 \mathrm{~h}$ integration starting at GPS-time $t_{0}=732490940 \mathrm{~s}$, the orbital velocity at the midpoint is $\vec{\beta}_{1}=[0.36,-9.13,-3.96] \times 10^{-5}$, and the corresponding family of circles is shown in figure 1 . Note that the family of circles only depends on the orbital velocity $\vec{\beta}_{1}$, and not on $f, f_{\mathrm{s}}$ and $\vec{n}_{\mathrm{s}}$, as can be seen in rewriting (34) as $\vec{\beta}_{1} \cdot \vec{n}=\beta_{1} \cos \theta$, where $\theta$ is the opening angle of the 'cone' with respect to the central direction $\vec{\beta}_{1}$, and the circles can therefore be parametrized by $\theta \in[0, \pi]$. This equation also determines the upper limit on the frequency mismatch $\Delta f$ for which such a local maximum is possible, namely, by rewriting (34) as

$$
\left|\frac{\Delta f}{f_{\mathrm{s}}}\right|=\frac{\left|\vec{\beta}_{1} \cdot \Delta \vec{n}\right|}{\left|1+\vec{\beta}_{1} \cdot \vec{n}\right|} \leqslant \frac{2 \beta_{1}}{1-\beta_{1}}=2 \beta_{1}+\mathcal{O}\left(\beta_{1}^{2}\right),
$$

we see that the maximal relative frequency mismatch is bounded by twice the Doppler shift due to the orbital velocity. Using the value $V_{\text {orb }} \approx 3 \times 10^{4} \mathrm{~m} \mathrm{~s}^{-1}$, this corresponds to a 'Doppler window' of $\Delta f / f \sim \pm 2 \times 10^{-4}$. 


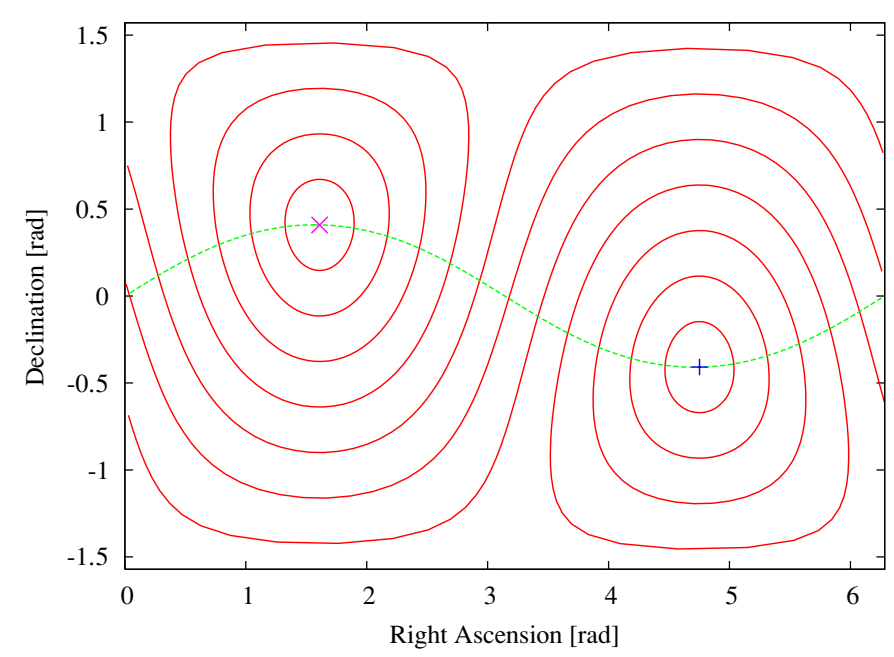

Figure 1. Family of circles described by (34) for a $T=10 \mathrm{~h}$ observation starting at GPS-time $t_{0}=732490940 \mathrm{~s}$. The direction of $\vec{\beta}_{1}$ is marked by ' $\times$ ', while ' + ' indicates the direction $-\vec{\beta}_{1}$. The circles shown correspond to the opening angles $\theta_{i}=\frac{i \pi}{N+1}$, where $N=11$ and $i=1, \ldots, N$. The dashed line indicates the ecliptic plane.

We note another interesting property of the approximate expression (29) for the matchedfiltering amplitude $|X|$ : namely, from the above discussion we know that the local maxima of $|X|$ occur on or close to the circles described by (34), which are characterized by $a_{1} \approx 0$. Therefore, we see from (29) that the amplitude of $\left|X_{1}\right|$ along these circles will be dominated by the factor $J_{n *}\left(K_{A}\right)$, where $K_{A}$ is proportional to $A_{\perp}$, the projection of $\vec{A}$ into the equatorial plane. This factor is therefore symmetrical in the target sky-position $\vec{n}$ with respect to the equatorial plane, a feature which can indeed be seen in the plot of (29) shown in figure 4(b). Interestingly, this symmetry can still be present in the fully numerical $\mathcal{F}$-statistic, as seen, for example, in figure $3(b)$, while the effects of spin motion and antenna patterns can also mask this feature depending on the parameters, as seen in figure 3(a).

\section{Comparison to fully numerical results for the $\mathcal{F}$-statistic}

We now turn to comparing these analytic results to the actual parameter-space structure of the fully numerical $\mathcal{F}$-statistic including all effects of amplitude modulation and using an ephemeris-based description of the detector motion. As an example, we consider a $10 \mathrm{~h}$ observation time starting at GPS-time $t_{0}=732490940 \mathrm{~s}$, and we use a fake pulsar signal at $f_{\mathrm{s}}=100 \mathrm{~Hz}$ and sky-position $\left(\alpha_{\mathrm{s}}, \delta_{\mathrm{s}}\right)=(2,1)$, for two different choices of amplitude parameters shown in table 1 . In the following, the detector location is always chosen to be the LIGO Livingston Observatory (LLO), and we only consider pure signals without noise. The fake pulsar signal is generated using makefakedata_v4, and the $\mathcal{F}$-statistic [5] is calculated using ComputeFStatistic ${ }^{1}$.

We observe the following structure of the $\mathcal{F}$-statistic over the parameter space $\{\alpha, \delta, f\}$ : searching over the sky at a perfectly matched target-frequency $f=f_{\mathrm{s}}$, the $\mathcal{F}$-statistic has a maximum at the signal sky-position $\left(\alpha_{\mathrm{s}}, \delta_{\mathrm{s}}\right)$, as expected, but remains of the same order on a very narrow, complete circle over the sky (obviously including the signal position),

1 These codes are found in LALApps[1], under src/pulsar/Injections and src/pulsar/FDS_isolated. 


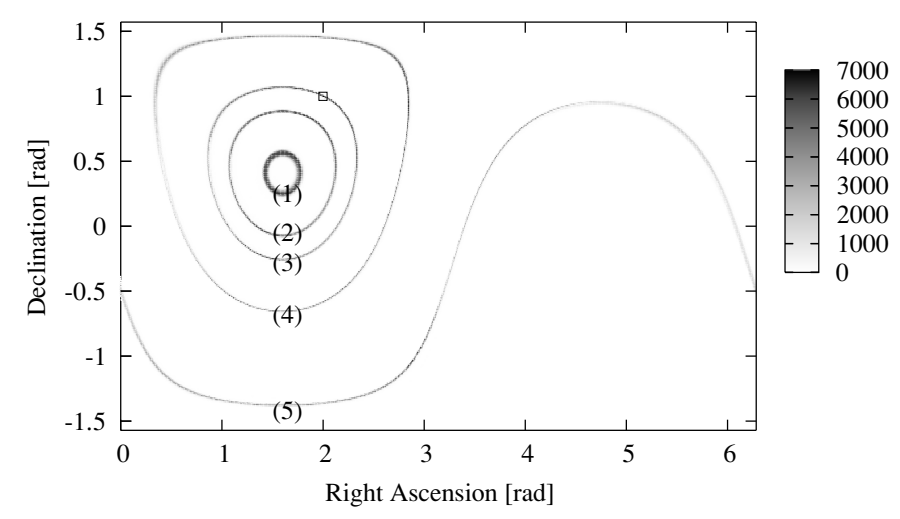

Figure 2. Plot of the $\mathcal{F}$-statistic over the sky for different target frequencies $f:(1) f=100.002 \mathrm{~Hz}$, (2) $f=100.001 \mathrm{~Hz}$, (3) $f=f_{\mathrm{s}}=100 \mathrm{~Hz}$, (4) $f=99.997 \mathrm{~Hz}$ and (5) $f=99.99 \mathrm{~Hz}$. The parameters of the injected signal correspond to model I in table 1, and the small square indicates the sky position of the signal.

Table 1. Amplitude and phase parameters of two fake pulsar signals.

\begin{tabular}{lllllll}
\hline Model & $f(\mathrm{~Hz})$ & $(\alpha, \delta)(\mathrm{rad})$ & $A_{+}$ & $A_{\times}$ & $\Psi$ & $\Phi_{0}$ \\
\hline I & 100 & $(2.0,1.0)$ & 1.0 & 0.5 & 1.0 & 2.0 \\
II & 100 & $(2.0,1.0)$ & 1.0 & 0.0 & 0.0 & 0.0 \\
\hline
\end{tabular}

while it is practically zero everywhere else. For mismatched target-frequencies $f$ (within the Doppler window of $f_{\mathrm{s}}$ ), there is a different but concentric circle of $\mathcal{F}$-values comparable to the maximum, with a larger radius for $f<f_{\mathrm{s}}$ and a smaller radius for $f>f_{\mathrm{s}}$, as shown in figure 2 . This agrees qualitatively and quantitatively surprisingly well with the circle structure predicted solely based on the effect of the orbital Doppler shift, i.e., (34) and figure 1.

In the next step, we run an all-sky search over the whole Doppler window $f \in$ $\left(1 \pm 2 \times 10^{-4}\right) f_{\mathrm{s}}$, corresponding to a dense superposition of these circles for the different target frequencies. The resulting $\mathcal{F}$-statistic projected on the sky is shown in figures $3(a)$ and $(b)$ for pulsar signals I and II, respectively. From these projected plots, we see more clearly that the variation of the amplitude of the $\mathcal{F}$-statistic maxima is more sensitive to the polarization parameters of the signal, due to effects of the antenna pattern. For example, figure 3(b) shows some of the equatorial symmetry characteristic for the approximate expression (29), as discussed in section 5 , in particular we see a mirror image in $(2,-1)$ of the absolute maximum at the signal position $(2,1)$. For signal I, on the other hand, the projected $\mathcal{F}$ is more asymmetrical and there is no mirror image of the maximum in the southern hemisphere.

Summarizing these observations we can state that the locations of the local maxima of $\mathcal{F}$ are dominantly determined by the orbital velocity of the earth, and are described very well ${ }^{2}$ by the circles (34), independently of detector location and amplitude parameters of the signal. The spin motion of the detector and amplitude modulation due to the rotating antenna pattern, on the other hand, are responsible for the variation of the amplitude of $\mathcal{F}$ along these circles, as seen in figure 3 .

2 A preliminary numerical comparison of the frequency $f_{\mathcal{F}}$ of the maximum of $\mathcal{F}$ for a fixed sky position $\vec{n}$ and the theoretical prediction $f_{\text {th }}$ from (34) yields the bound $\left|f_{\mathcal{F}}-f_{\text {th }}\right| / f_{\mathcal{F}}<10^{-6}$ over the whole sky. 
(a)

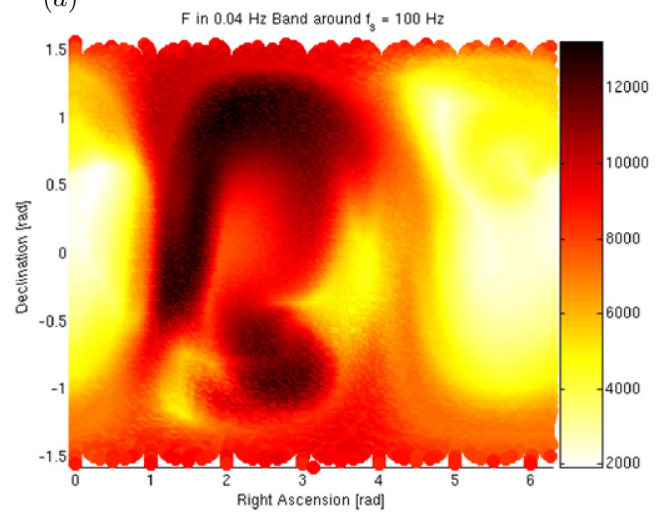

(b)

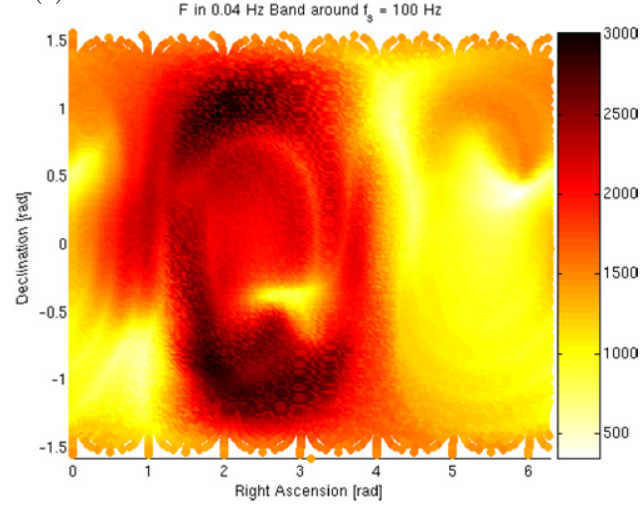

Figure 3. Projected $\mathcal{F}$-statistic over the sky in the frequency range $f \in\left(1 \pm 2 \times 10^{-4}\right) f_{\mathrm{s}}$ for (a) pulsar signal I and (b) signal II as defined in table 1.

(a)

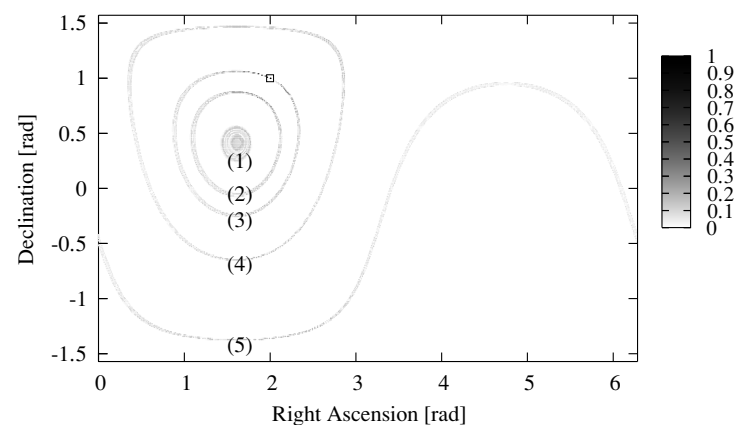

(b)

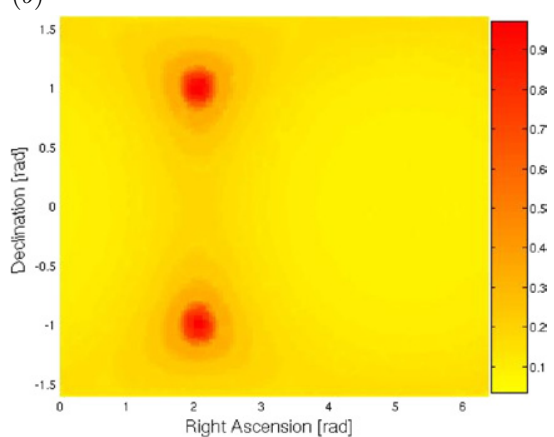

Figure 4. The approximate matched-filtering amplitude $\left|X_{1}\right|^{2}$ of model I in table 1 . These plots show $\left|X_{1}\right|^{2}$ over the sky for (a) different target frequencies (1) $f=100.002 \mathrm{~Hz}$, (2) $f=$ $100.001 \mathrm{~Hz}$, (3) $f=f_{\mathrm{s}}=100 \mathrm{~Hz}$, (4) $f=99.997 \mathrm{~Hz}$ and (5) $f=99.99 \mathrm{~Hz}$, and (b) over the whole Doppler window $f \in\left(1 \pm 2 \times 10^{-4}\right) f_{\mathrm{s}}$ projected on the sky. The small square indicates the sky position of the signal.

Finally, we plot the approximate detection amplitude $\left|X_{1}\right|^{2}$ given by (29) over the sky for different target frequencies, as shown in figure 4(a), which shows some striking similarities to the 'exact' result in figure 2. In particular, it exhibits the feature of vanishing very rapidly outside the narrow 'circles' described approximately by (34). The amplitude of $\left|X_{1}\right|^{2}$ on the circles, however, is not a good description of the corresponding $\mathcal{F}$-statistic amplitude, as $\left|X_{1}\right|^{2}$ is much more strongly peaked around the signal location and decreases rather rapidly to about 0.1 on most of the circles (see figure $4(b)$ ), while the $\mathcal{F}$-statistic has a similar amplitude to the maximum over most of the circles. Note also that figure $4(b)$ illustrates the equatorial symmetry of the projected $\left|X_{1}\right|^{2}$ as discussed at the end of section 5 .

\section{Discussion}

In a practical search for continuous gravitational waves we will usually be interested in the highest values ('candidates') of $\mathcal{F}$ over a chosen volume of the parameter space. From the 
results presented here we expect that a real signal would generate candidates nearly everywhere on the sky within the 'double' Doppler window $\pm 2 \times 10^{-4}$ of the signal frequency $f_{\mathrm{s}}$. All these candidates, however, will satisfy (34) with an (approximately) identical value on the right-hand side, and so the quantity $\kappa \equiv f\left(1+\vec{\beta}_{1} \cdot \vec{n}\right)$ is an (approximate) invariant of candidates caused by the same signal. This could be a very useful criterion for classifying equivalent candidates, and also for determining coincident candidates from different detectors. One could further use this to construct coincidence tests of candidates from different observation times, as $\vec{\beta}_{1}(t)$ is a known function of time. In order for these methods to be practically useful, however, the errors of the different approximations need to be quantified, and we need to test the robustness of the suggested coincidence criteria in the presence of noise. Further work is also required in order to quantify the agreement between the different levels of approximation (29) and (35) and the exact numerical results. The width of these circles is expected to be well described by the local metric, but this relation also needs to be studied and quantified in more detail.

\section{Acknowledgments}

We would like to thank Badri Krishnan, Maria Alessandra Papa, Xavier Siemens and Alicia Sintes for helpful discussions.

\section{References}

[1] LALApps, A collection of software tools for data-analysis (using LAL) http://www.lsc-group.phys.uwm. edu/daswg/projects/lalapps.html

[2] Abbot B et al (LIGO Scientific Collaboration) 2004 Phys. Rev. D 69082004

[3] Abbot B et al (LIGO Scientific Collaboration) 2005 Phys. Rev. Lett. 94181103 (Preprint gr-qc/0410007)

[4] Brady P R, Creighton T, Cutler C and Schutz B F 1998 Phys. Rev. D 57 2101-16

[5] Jaranowski P, Królak A and Schutz B F 1998 Phys. Rev. D 58063001

[6] Jotania K, Valluri S R and Dhurandhar S V 1996 Astron. Astrophys. 306 317-25

[7] Krishnan B, Sintes A M, Papa M, Schutz B F, Frasca S and Palomba C 2004 Phys. Rev. D 70802001

[8] Owen B J 1996 Phys. Rev. D 53 6749-61

[9] Astone P et al 2003 Class. Quantum Grav. 20665

[10] Valluri S R, Drozd J J, Chishtie F A, Biggs R G, Davison M, Dhurandhar S V and Sathyaprakash B S 2002 Class. Quantum Grav. 19 1327-34 